\title{
Analysis of Hole Transport in Thin Films and Nanoparticle Assemblies of Poly(3-hexylthiophene)
}

\author{
Xu Han ${ }^{(1)}$, Monojit Bag ${ }^{(2)}$, Timothy S. Gehan ${ }^{(2)}$, Dhandapani Venkataraman ${ }^{(2)}$, and \\ Dimitrios Maroudas ${ }^{(1)}$ *
}

\begin{abstract}
We report numerical simulation results on hole transport in layers of the organic polymer poly(3-hexylthiophene) (P3HT) of different nanostructures based on a deterministic, phenomenological drift-diffusion-reaction model that accounts for hole trapping-detrapping kinetics. The model is used to characterize the various P3HT layers examined in terms of their hole transport dispersivity. The model reproduces well experimental data of photocurrent evolution in P3HT samples ranging from drop cast thin films to surfactant-stabilized nanoparticle assemblies, explains the role of excess surfactant molecules in hole trapping for assemblies of P3HT nanoparticles, and demonstrates quantitatively the potential of using nanoparticle assemblies in organic photovoltaic devices.
\end{abstract}

Keywords: charge transport modeling, charge trapping, organic photovoltaics, nanoparticle assemblies 


\section{Introduction}

Recently, there has been a growing interest in using conjugated polymer nanoparticles [1] for the fabrication of organic active layers that are used in light-emitting diodes, organic solar cells, field-effect transistors, and other optoelectronic devices [2-4]. Using nanoparticles has the significant advantage that individual electron-donor and electron-acceptor particles of tunable size and internal structure can be pre-formed and then self-assembled into well-defined activelayer morphologies [5]. Moreover, using printing and coating methods, nanoparticles can be formulated as aqueous dispersions for large-area manufacturing making them attractive for active layer fabrication in organic photovoltaic cells. An important feature of conjugated polymer nanoparticles is that they are stabilized by surfactants, which are expected to introduce trapping sites for electrons and holes and impede charge transport. Yet, the observed hole mobility in surfactant-stabilized conjugated nanoparticle assemblies is comparable to that of pristine films [6]. In addition to charge mobility, charge trapping and detrapping also play an important role in the overall performance of these films as photovoltaic active layers. Herein, we address the questions of hole mobility and trapping through comparisons of the computed charge transport coefficients and trapping-detrapping kinetics in nanoparticle assemblies and pristine thin films of the electron-donor conjugated polymer poly(3-hexylthiophene) (P3HT) based on computer simulations of time-of-flight (TOF) experiments for measuring transient photocurrents in these P3HT layers.

Computational modeling is a powerful means to further our understanding of charge transport in organic polymer films. Dynamic Monte Carlo simulations have been used to understand mechanisms of charge carrier generation and transport $[7,8]$. Numerical simulations informed about such mechanisms have described satisfactorily physical properties of such 
organic layers [9]. Deterministic, phenomenological drift-diffusion-reaction models also have managed to capture transient photocurrents measured in bulk-heterojunction organic photovoltaic (OPV) devices [10-12]. The computational efficiency of such models, especially within the effective-medium approximation [12], is particularly appealing for the simulation of OPV device operation and the optimization of OPV device performance. Such deterministic models, however, have not been used to model charge transport in OPV active layers consisting of nanoparticle assemblies, where incorporating the kinetics of trapping and detrapping of charge carriers into the models is particularly important.

In this study, we examine hole transport dispersivity in thin films and nanoparticle assemblies of P3HT and explore the effects of surfactant on hole trapping. The purpose of this Letter is the development and validation of a deterministic, phenomenological drift-diffusionreaction model for hole transport in $\mathrm{P} 3 \mathrm{HT}$ thin films and nanoparticle assemblies that can reproduce experimental data for photocurrent evolution in samples of these materials with different nanostructures and over a range of applied voltages. The model accounts for the kinetics of hole trapping and detrapping in the various $\mathrm{P} 3 \mathrm{HT}$ films and is used to characterize the corresponding nanostructures in terms of their hole transport dispersivity, to explain the role of excess surfactant molecules in hole trapping for assemblies of P3HT nanoparticles, and to explore the potential of using nanoparticle assemblies as active layers in OPV devices.

\section{Experimental Methods, Model, and Computational Methods}

The experimental procedures followed for sample preparation and characterization and for the measurement of transient photocurrents were presented in Ref. 6. P3HT thin films were drop cast on indium tin oxide (ITO) substrates. Some of the nanoparticle assemblies were 
prepared after centrifugal filtration of the dispersion in order to remove excess surfactant molecules. Both non-centrifuged and centrifuged nanoparticle assemblies were spray coated on the ITO substrates. A thin aluminum (Al) film was then thermally deposited on the P3HT layers. The Al electrode was positively biased with respect to the ITO electrode. 355 -nm pulsed ( $\sim 10$ ns) laser illumination was applied near the Al electrode. The TOF method was used to determine hole mobilities [6]. Systematic experiments on various samples under different applied biases demonstrated hole mobility in assemblies of surfactant surrounded nanoparticles comparable with those of drop cast P3HT thin films. It was also found that the presence of excess surfactant in P3HT nanoparticle assemblies increased their hole transport dispersivity [6].

For a quantitative analysis of hole transport in the above three types of thin films and nanoparticle assemblies, we have developed a comprehensive one-dimensional (1D) transient drift-diffusion-reaction model. The model accounts for hole trapping and detrapping in addition to hole diffusion and drift and consists of coupled boundary-value problems (BVPs) for the evolution of the free and trapped hole density fields in the polymer layer and for the electrostatic potential distribution in the material, governed by the following equations:

$$
\begin{aligned}
& \frac{\partial p_{f}}{\partial t}=\frac{1}{e} \frac{\partial}{\partial x}\left(\mu_{p} k_{B} T \frac{\partial p_{f}}{\partial x}-\mu_{p} p_{f} e E\right)-\frac{\partial p_{t}}{\partial t}, \\
& \frac{\partial p_{t}}{\partial t}=C_{t}\left(P_{t}-p_{t}\right) p_{f}-C_{d t} p_{t}, \\
& \text { and } \quad \frac{\partial^{2} \Phi}{\partial x^{2}}=-\frac{e\left(p_{f}+p_{t}\right)}{\varepsilon_{0} \varepsilon} .
\end{aligned}
$$

Equation (1) describes the evolution of the free hole density $p_{f}(x, t)$, where $p_{t}(x, t)$ is the trapped hole density. In Eq. (1), $t, e$, and $x$ denote time, elementary charge, and the coordinate along the film thickness direction and perpendicular to the electrodes, respectively, $k_{B}$ is Boltzmann's 
constant, $T$ is temperature, and $E$ is the magnitude of the electric field, $\mathbf{E}=E \mathbf{e}_{x}$, directed along $x$. The hole mobility, $\mu_{p}$, is taken to follow the Poole-Frenkel relation [13], $\mu_{p}=\mu_{0} \exp \left(\gamma|E|^{1 / 2}\right)$, with $\mu_{0}$ and $\gamma$ being the zero-field mobility and the field dependence coefficient, respectively. For the free holes, boundary conditions of thermionic injection with image charge effects [14] were applied for the hole fluxes at the polymer/electrode interfaces, $x=0$ and $x=L$. Equation (2) expresses the kinetics of hole trapping and detrapping with the initial condition that no generated charges are trapped, $p_{t}(x, t=0)=0 ; C_{t}$ and $C_{d t}$ are the trapping and detrapping kinetic rate coefficients, respectively, and $P_{t}$ is the density of trapping sites in the polymeric material. Trapping-detrapping kinetics according to Eq. (2) assumes a single trapping energy level. Equation (3) is Poisson's equation for the electrostatic potential $\Phi, E=-\partial \Phi / \partial x, \varepsilon_{0}$ is the vacuum permittivity and $\varepsilon$ is the relative permittivity of the material; $p \equiv p_{f}+p_{t}$ is the total density of holes. Dirichlet boundary conditions for $\Phi$ were applied at $x=0$ and $x=L$, as determined by the applied potentials at the two electrodes. As initial condition for the free hole density, we use an exponentially decaying (from $x=0$ ) distribution, $p_{f}(x, 0)=P_{0} \exp (-x / \lambda)[15]$, where $\lambda$ is on the order of the penetration depth of the exciting light.

We solved the coupled BVPs governed by Eqs. (1)-(3) numerically using the finitedifference method for domain discretization. To avoid numerical instabilities in steep propagating charge fronts caused by strong electric fields, we employed upwinding [16] for the computation of the drift flux in Eq. (1). The convergence of the numerical results with refining the finite-difference grid resolution was ensured by using fine grids in the numerical simulations (with as many as $n=6000$ grid points). We implemented the implicit Euler method for time stepping Eqs. (1) and (2) and calculated the transient photocurrent $I(t)$, by integrating the local free hole flux (current density) over the domain and averaging, according to the equation 


$$
I(t)=A \frac{1}{n} \sum_{i=1}^{i=n} j_{i}=\frac{1}{n} A \mu_{0} \sum_{i=1}^{i=n} \exp \left(\gamma \sqrt{\left|E_{i}\right|}\right)\left(-k_{B} T \frac{\partial p_{f i}}{\partial x}+p_{f i} e E_{i}\right),
$$

where $A$ is the film cross-sectional area (contact area with the electrodes) and $j$ is the current density. For simplicity, Eq. (4) assumes a uniform grid of $n$ points (nodes " $i$ "); the subscripts $i$ in the quantities of the right-hand sides of Eq. (4) denote the nodal values of the quantities.

\section{Results and Discussion}
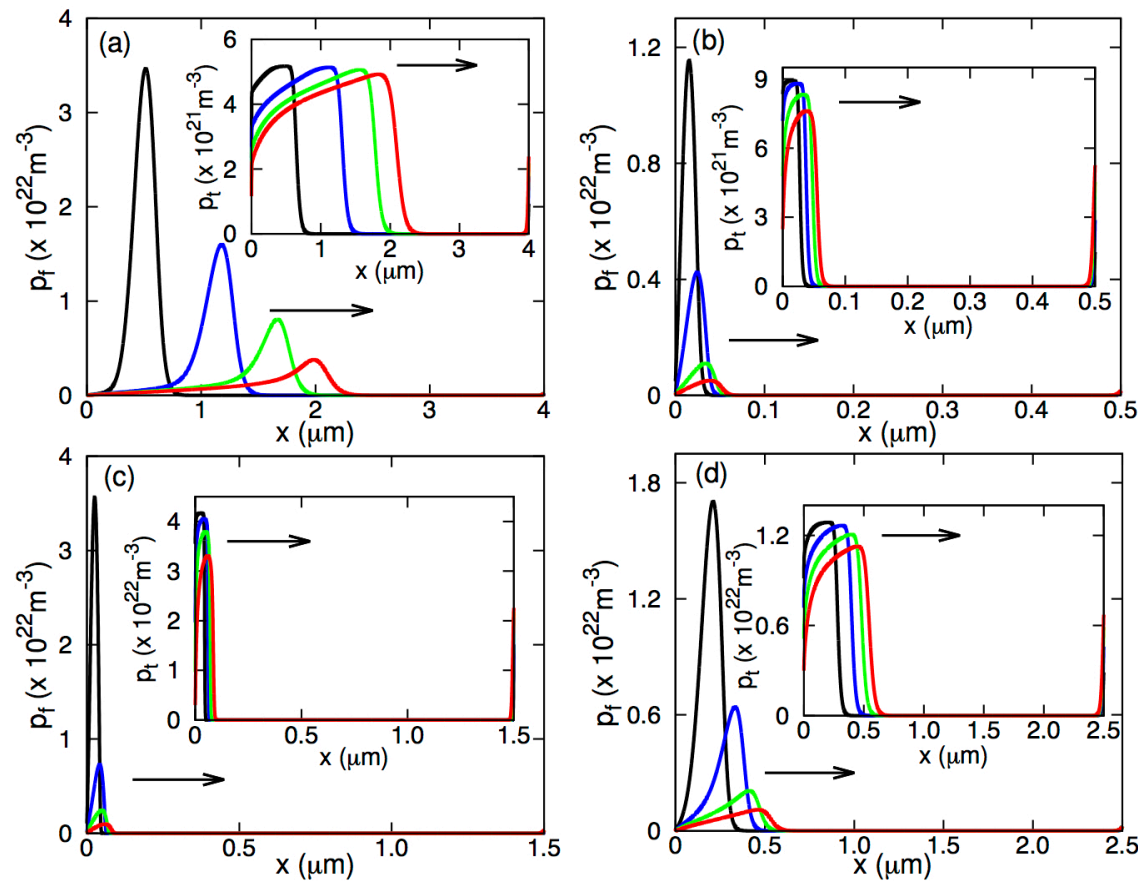

FIG. 1: Simulated evolution (with time as indicated by the arrows) of free and trapped (insets) hole density profiles in P3HT sample (a) P1 under bias of $9 \mathrm{~V}$, (b) P2 under $2 \mathrm{~V}$, (c) P3 under 4 $\mathrm{V}$, and (d) P4 under $4 \mathrm{~V}$. The corresponding time instants for the profiles shown are (a) 19, 47, 71, and $90 \mu \mathrm{s}$; (b) 1.6, 3.7, 9.0, and $19 \mu \mathrm{s}$; (c) 4.8, 14, 31, and $96 \mu \mathrm{s}$; and (d) 23, 45, 78, and 129 $\mu \mathrm{s}$. For the simulations, the values for the transport coefficients, kinetic parameters, and material properties used are within the range of the fitting parameter values reported in Table 1.

Before comparing the predictions of our model with the experimentally measured transient photocurrents in our P3HT samples, we validated the model by fitting its predictions to the experimental $I(t)$ data from the TOF measurements of Ref. 13 for nondispersive hole transport in the polyfluorene copolymer poly[(9,9-dioctylfluorenyl1-2,7-diyl)-co-(4,4'-(N-(4-sec- 
butylphenyl))diphenylamine)]. The model reproduced the data successfully throughout the duration of the TOF experiment, including the initial increase, plateau region, and tail region of the transient photocurrent [13]. Having validated the model, we solved the coupled BVPs governed by Eqs. (1)-(3) and fitted our experimental $I(t)$ measurements for our P3HT samples, thus obtaining as fitting parameters hole transport properties, kinetic coefficients, and material properties for the different nanostructured P3HT films used in the TOF experiments.

We modeled hole transport under various applied biases in a 4- $\mu \mathrm{m}$-thick P3HT drop-cast thin film (sample P1), thin films of non-centrifuged nanoparticle assemblies with excess surfactant molecules of thickness equal to $0.5 \mu \mathrm{m}$ (sample P2) and $1.5 \mu \mathrm{m}$ (sample P3), and a $2.5-\mu \mathrm{m}$-thick film of centrifuged nanoparticle assemblies with the excess surfactant molecules removed (sample P4). We found that hole transport dynamics is significantly different in these films of different nanostructures. Figures 1(a)-(d) show the evolution of the free hole density profiles in these four samples under certain applied biases; in each figure, the inset depicts the evolution of the corresponding trapped hole density profile. It is evident that trapping and detrapping of charge carriers due to structural defects in the amorphous layer distort the charge density profile from a Gaussian distribution [17]. The peak locations of the free and trapped hole packets propagate in the polymeric materials of our samples at speeds determined by the hole mobility and the electric field strength. In P1, Fig. 1(a), the overall hole distribution is skewed and tail regions are formed on its back since trapped holes cannot escape from their trap states fast enough to catch up with the free carriers; once these holes are released (detrapped), they contribute to the free hole density. However, in P2 and P3, Figs. 1(b) and 1(c), respectively, the distortion of the free and trapped hole density distributions from Gaussians is much less pronounced than that in P1, which indicates that these two samples are characterized by a much 
faster hole detrapping rate. In addition, the peaks of these hole packets move slower than that in P1, suggesting lower hole mobilities for these two samples. Moreover, the peaks of the hole distributions in $\mathrm{P} 2$ and $\mathrm{P} 3$ are lowered much faster than that of $\mathrm{P} 1$, which is expected to result in a faster decrease in the generated transient photocurrent. Finally, in P4, Fig. 1(d), the hole distributions are skewed Gaussians with fronts for both free and trapped holes propagating and exhibiting subtle tails for the free holes and almost rectangular-shaped tails for the trapped holes, similar to those of P1, implying a slow detrapping rate for this sample too.
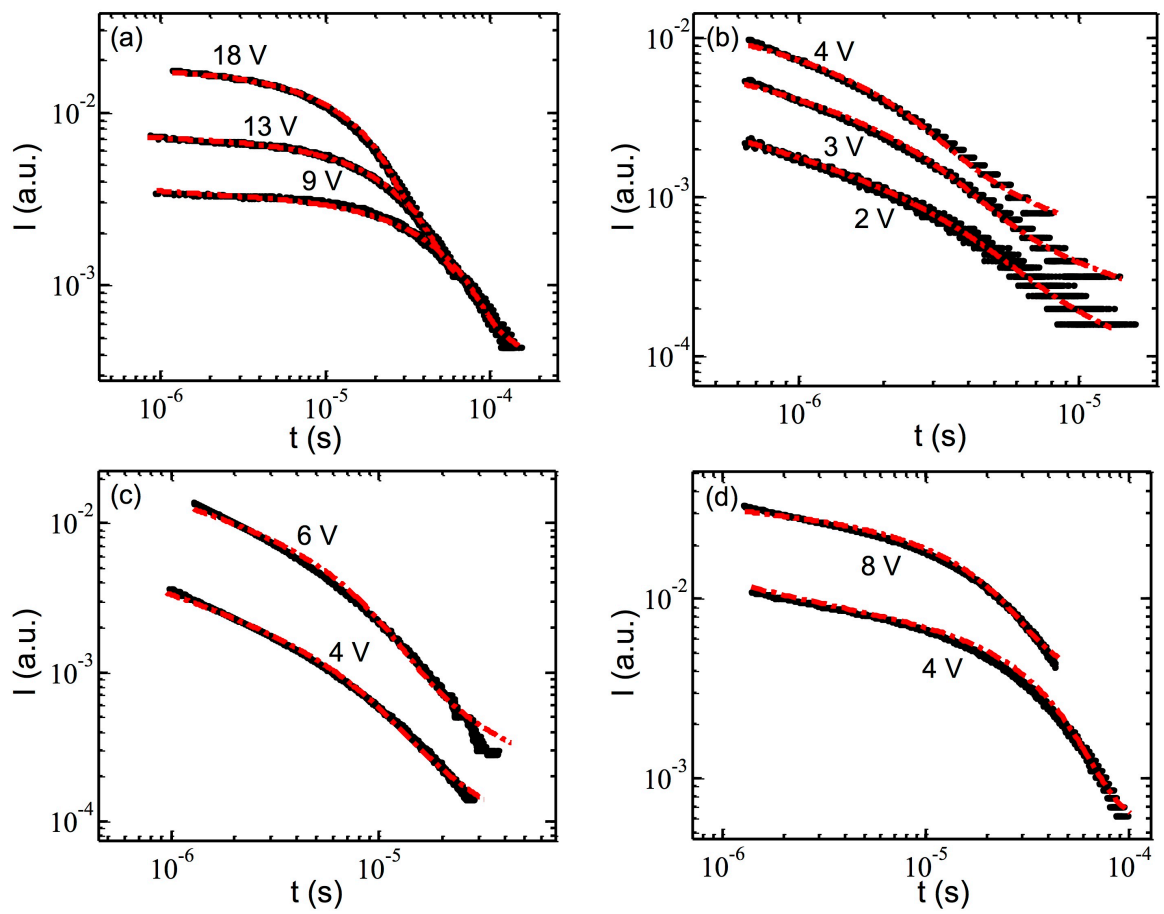

FIG. 2: Best fits of simulation predictions (red dot-dashed lines) to experimental data (black solid circles) for photocurrent evolution $I(t)$ under various applied biases in P3HT samples (a) $\mathrm{P} 1$, (b) P2, (c) P3, and (d) P4. For every film, the contact area with the electrodes is $A=6 \mathrm{~mm}^{2}$.

We have fitted the predictions of numerical simulations according to the hole transport model governed by Eqs. (1)-(3) to the experimental measurements of transient photocurrent $I(t)$ in the four samples $\mathrm{P} 1, \mathrm{P} 2, \mathrm{P} 3$, and $\mathrm{P} 4$ with the zero-field mobility $\mu_{0}$, kinetic parameters $C_{t}$ and $C_{d t}$, and material properties $P_{0}$ and $P_{t}$ treated as fitting parameters. The field-dependence 
coefficient $\gamma$ was determined by fitting simulation predictions to experimental data under different applied voltages. Figure 2 compares simulation results with experimental data for photocurrent evolution, $I(t)$, presented in $\log -\log$ plots by showing best fits of simulation predictions (red dot-dashed lines) to the experimental data (black solid circles) for the four P3HT samples, P1, P2, P3, and P4, under various applied biases. The comparison focuses on the time period after $\sim 0.5 \mu$ s from the initiation of the experiment, since this initial transient stage is dominated by the discharge current at the polymer-electrode interface as opposed to charge carrier transport in the P3HT layer. In each case, the simulation results reproduce successfully the measured transit time, which is a metric of the characteristic time for the majority of the holes to reach the anode and is determined experimentally as the time corresponding to the intersection of the two linear regions in the logarithmic (log-log) plot of photocurrent evolution. Moreover, the simulation results capture successfully the entire dynamical behavior of the charge carriers in the examined P3HT materials of different nanostructure and reproduce the experimental transient photocurrent data very well for all samples and applied voltages, which validates further our model based on Eqs. (1)-(3). It is evident from Fig. 2 that the slopes of the first linear regions of their photocurrent transients in P2 and P3, Figs. 2(b) and 2(c), are much steeper than those for P1 and P4 that are much closer to zero, Figs. 2(a) and 2(d). These differences in the $I(t)$ slopes in the first linear regions of the corresponding log-log plots reflect the faster rates of decrease in the hole densities over a short time period for samples P2 and P3 exhibited in Figs. 1(b) and 1(c), respectively, compared to those for P1 and P4 in Figs. 1(a) and 1(d), respectively. In brief, the results of Figs. 1 and 2 indicate clearly that hole transport is much more dispersive in samples $\mathrm{P} 2$ and $\mathrm{P} 3$ than in samples P1 and P4. 
TABLE 1. Hole transport coefficients, kinetic parameters, and material properties derived from fitting modeling predictions to experimental data for transient photocurrent $I(t)$ in the P3HT layer samples P1, P2, P3, and P4.

\begin{tabular}{ccccc}
\hline \hline Sample & $\mathrm{P} 1$ & $\mathrm{P} 2$ & $\mathrm{P} 3$ & $\mathrm{P} 4$ \\
\hline$P_{0}\left(\times 10^{25} \mathrm{~m}^{-3}\right)$ & $2.4-3.65$ & $1.6-2.6$ & $3.6-8.0$ & $2.6-2.8$ \\
$P_{t}\left(\times 10^{22} \mathrm{~m}^{-3}\right)$ & $0.52-1.0$ & $1.0-1.8$ & $4.2-9.6$ & $1.24-1.3$ \\
$C_{t}\left(\times 10^{-16} \mathrm{~m}^{3} \mathrm{~s}^{-1}\right)$ & $0.55-0.6$ & $8-10$ & 7.5 & 0.6 \\
$P_{t} C_{t}\left(\times 10^{6} \mathrm{~s}^{-1}\right)$ & $0.29-0.6$ & $10-18$ & $31.5-72$ & $0.74-0.78$ \\
$\mu_{0}\left(\times 10^{-5} \mathrm{~cm}^{2} \mathrm{~V}^{-1} \mathrm{~s}^{-1}\right)$ & 6.5 & 0.9 & 0.9 & 3.7 \\
$\gamma\left(\times 10^{-4}\left(\mathrm{~V} / \mathrm{m}^{-1 / 2}\right)\right.$ & $3.9-4.6$ & $3.0-3.2$ & $3.2-3.7$ & $3.2-3.6$ \\
$C_{d t}\left(\times 10^{4} \mathrm{~s}^{-1}\right)$ & $1.0-2.2$ & $10-19$ & $20-42$ & $1.1-1.7$ \\
$d p_{t} / d t\left(\times 10^{25} \mathrm{~m}^{-3} \mathrm{~s}^{-1}\right)$ & $2.36-5.23$ & $9.25-40.4$ & $8.1-30.1$ & $3.1-6.0$ \\
\hline \hline
\end{tabular}

The computed fitting parameters and derived trapping rate metrics based on these parameters are listed in Table 1 for all four samples. The detailed determination of these parameters follows the procedure of Ref. 12. In all cases, the parameters vary over narrow ranges under different applied biases, but the trends are very clear in comparing the various samples with respect to their hole transport dispersivity. Parameter variations with different applied bias are expected [18-20]; this is because the applied bias affects the initial free charge carrier generation from exciton dissociation and, therefore, the occupation of trap states, the trapping and detrapping rates, and the field dependence of carrier mobility. It is evident that the abilities of the different nanostructured P3HT films to generate free charges, as expressed by parameter $P_{0}$, are comparable. On the other hand, the density of trapping sites $P_{t}$ varies with 
varying nanostructure. Sample $\mathrm{P} 4$ has a slightly increased value of $P_{t}$ than that in P1; this value is further increased in $\mathrm{P} 2$ and is much higher for the thicker film P3. Comparing the $P_{t}$ values of samples P2, P3, and P4, we conclude that the excess surfactant molecules surrounding P3HT nanoparticles introduce additional hole trapping sites in the organic layers, which is enhanced with increasing film thickness, and reduce the corresponding packing density. The trapping rate coefficient $C_{t}$ is found to be higher by more than one order of magnitude in non-centrifuged nanoparticle assemblies (samples P2 and P3) than in the other two nanostructured films (samples $\mathrm{P} 1$ and $\mathrm{P} 4)$. The resulting values of the trapping rate metric given by the product $P_{t} C_{t}$ imply that the charge trapping effect is much (up to two orders of magnitude) stronger in non-centrifuged nanoparticle assemblies (samples P2 and P3) than in drop cast thin films and centrifuged nanoparticle assemblies (samples P1 and P4, respectively). These findings explain the similar hole transport dispersivity in samples P1 and P4 and the much more dispersive hole transport in samples P2 and P3, as observed in the results of Figs. 1 and 2. The zero-field hole mobilities are within a factor 2 in the drop cast thin film (P1) and the centrifuged nanoparticle assembly (P4). These mobility values are substantially lower in the non-centrifuged samples (P2 and P3). The field dependence of the hole mobility, expressed by $\gamma$, is roughly the same for all the P3HT samples studied; this implies that the balancing effects between energetic and spatial disorder $[21,22]$ do not change substantially in the different samples that we examined. However, the detrapping rate coefficient $C_{d t}$ varies widely (over one order of magnitude) over different nanostructures; it is of comparable value in the drop cast thin film (P1) and the centrifuged nanoparticle assemblies (P4), but it is substantially higher in the non-centrifuged nanoparticle assembly samples (P2 and P3). 
It is known that the stabilizing surfactants that surround the nanoparticles introduce barriers for charges to tunnel through and hop to charge transport sites in neighboring nanoparticles [23]. From the results of Table 1, we conclude that the presence of excess surfactant molecules in nanoparticle assemblies has led to the introduction of more defects and an increased interparticle spacing, which result in higher trapping rates and lower hole mobilities. However, these trap states are characterized by shallow trapping energy levels and low activation energy barriers so that the trapped charge carriers can be detrapped easily to contribute to free charge carriers. Upon removal of the excess surfactants in centrifuged nanoparticle assemblies, the gaps between nanoparticles in the assemblies are reduced resulting in a highly improved hole mobility. Finally, for all samples, we computed the net trapping rate $d p_{t} / d t$, defined as the difference between the trapping and detrapping rates according to Eq. (2) at the transit time, integrated and averaged over the layer thickness. According to this metric, it is evident that the overall hole transport dispersivities in drop cast thin films (P1) are comparable with those in centrifuged nanoparticle assemblies (P4), while they are almost one order of magnitude stronger in non-centrifuged nanoparticle assemblies (P2 and P3).

\section{Conclusions}

In summary, we carried out a numerical simulation study of hole transport in P3HT films of different nanostructures based on a drift-diffusion-reaction model that accounts for hole trapping and detrapping kinetics. Our simulations reproduced experimental data for photocurrent transients and determined material and kinetic properties that govern hole transport in the $\mathrm{P} 3 \mathrm{HT}$ films. We found the hole transport properties of P3HT nanoparticle assemblies without excess surfactant to be comparable with those of drop-cast P3HT thin films and elucidated the role of excess surfactant in hole trapping. The charge carrier trapping and detrapping kinetics 
emphasized in our study have important implications for the design of OPV active layers, since space charge formation mediated by such trapping and detrapping can decrease internal electric fields and, therefore, promote bimolecular recombination and passivate charge separation. Our analysis demonstrates quantitatively the potential of using organic nanoparticles in developing active layers for OPV devices; this has been shown most clearly by the high zero-field mobility and the low net hole trapping rate of $\mathrm{P} 3 \mathrm{HT}$ nanoparticle assemblies with the excess surfactant molecules removed, quantities that were found to be comparable with those of pristine $\mathrm{P} 3 \mathrm{HT}$ thin films. The use of polymeric nanoparticle assemblies in the fabrication of OPV active layers introduces important control parameters, such as nanoparticle size and internal aggregate structure, to tune band structure and optimize charge transport efficiency. Analogous modeling results that reproduce experimental measurements of electron transport in poly-(benzothiadiazole vinylene) thin films and parameterize electron kinetics in such organic layers will be reported in a forthcoming publication.

\section{Acknowledgement}

This work was supported by Polymer-Based Materials for Harvesting Solar Energy, an Energy Frontier Research Center funded by the U.S. Department of Energy (DOE), Office of Basic Energy Sciences under Award No. DE-SC0001087. 


\section{References}

1. J. A. Labastide, M. Baghgar, I. Dujovne, Y. P. Yang, A. D. Dinsmore, B. G. Sumpter, D. Venkataraman, M. D. Barnes, J. Phys. Chem. Lett. 2 (2011) 3085.

2. A. R. Brown, C. P. Jarrett, D. M. de Leeuw, M. Matters, Synth. Mat. 88 (1997) 37.

3. G. G. Malliaras, R. H. Friend, Physics Today 58 (2005) 53.

4. A. Hagfeldt, M. Grätzel, Acc. Chem. Res. 33 (2000) 269.

5. D. Venkataraman, S. Yurt, B. H. Venkatraman, N. Gavvalapalli, J. Phys. Chem. Lett. 1 (2010) 947.

6. M. Bag, T. S. Gehan, D. D. Algaier, F. Liu, G. Nagarjuna, P. M. Lahti, T. P. Russell, D. Venkataraman, Adv. Mater. 25 (2013) 44.

7. H. Bassler, Phys. Status Solidi B 175 (1993) 15.

8. R. Noriega, A. Salleo, A. J. Spakowitz, Proc. Natl. Acad. Sci. U.S.A. 110 (2013) 16315.

9. E. Knapp, R Hausermann, H. U. Schwarzenbach, B. Ruhstaller, J. Appl. Phys. 108 (2010) 054504.

10. J. A. Barker, C. M. Ramsdale, N. C. Greenham, Phys. Rev. B 67 (2003) 075205.

11. C. R. McNeill, N. C. Greenham, Appl. Phys. Lett. 93 (2008) 203310.

12. I. Hwang, C. R. McNeil, N. C. Greenham, J. Appl. Phys. 106 (2009) 094506.

13. H. H. Fong, A. Papadimitratos, G. G. Malliaras, Appl. Phys. Lett. 89 (2006) 172116.

14. J. C. Scott, G. G. Malliaras, Chem. Phys. Lett. 299 (1999) 115.

15. H. Seki, J. Appl. Phys. 43 (1972) 1144.

16. B. A. Finlayson, Nonlinear Analysis in Chemical Engineering (McGraw-Hill, New York, 1980).

17. H. Scher, Phys. Rev. B 12 (1975) 2455.

18. J. Staudigel, M. Stossel, F. Steuber, J. Simmerer, J. Appl. Phys. 86 (1999) 7.

19. J. Guo, H. Ohkita, H.Benten, S. Ito, J. Am. Chem. Soc. 131 (2009) 16869. 
20. W. F. Pasveer, J. Cottaar, C. Tanase, R. Coehoorn, P. A. Bobbert, P. W. M. Blom, D. M. de Leeuw, M. A. J. Michels, Phys. Rev. Lett. 94 (2005) 206601.

21. A. J. Mozer, N. S. Sariciftci, Chem. Phys. Lett. 389 (2004) 438.

22. W. D. Gill, J. Appl. Phys. 43 (1972) 5033.

23. J. Kim, N. A. Kotov, Chem. Mater. 26 (2014) 134. 


\section{Graphical Abstract}
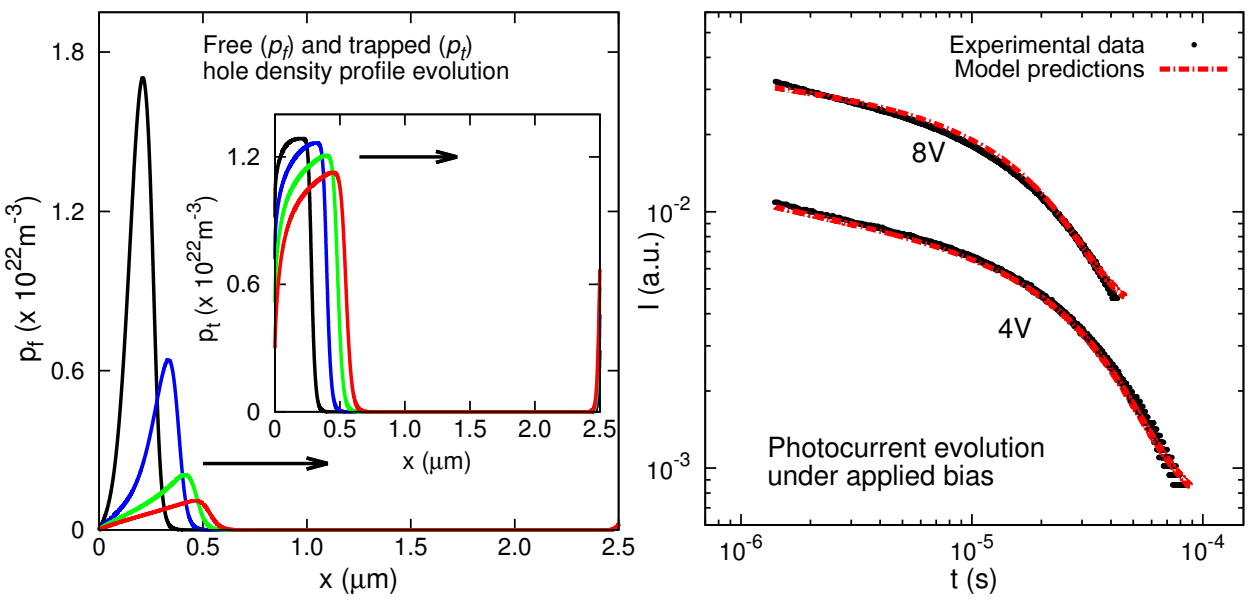